\title{
A Jewelry Modeler for the Fret-worked Bangles
}

\author{
Vishal Gulati \\ GJ University of Science and \\ Technology, Hisar, India
}

\author{
Puneet Tandon \\ PDPM-Indian Institute of Information \\ Technology, Design and \\ Manufacturing, Jabalpur, India
}

\author{
Hari Singh \\ National Institute of Technology \\ Kurukshetra, India
}

\begin{abstract}
A parametric feature based jewelry modeler for designing and manufacturing of the Fret-worked bangles. These bangles are made from gold and adorned with fretwork designs. Fretwork is a decorative design that is either carved in low relief on a solid background, or cut out to adorn a jewelry item. Fretwork designs are viewed as features that are to be recurrently removed from the stock-solid bangles. The jewelry modeler supports diverse integrated manufacturing through computer controlled manufacturing. Fret-worked bangles are fabricated using rapid prototyping technique.
\end{abstract}

\section{Keywords}

Jewelry modeler, Bangles, Parametric Feature Based

\section{INTRODUCTION}

Bangles are part of traditional jewelry, usually worn by women on each arm. In India, bangles made from gold have a religious connotation and a deep social significance. Mostly for an enhanced appeal, solid gold bangles have been studded with precious diamonds, gems and pearls or carved in low relief with decorative designs. These styles of exquisite bangles are very expensive because of having precious stones and heavy weight. This work produces a style of light-weight bangles, called Fretworked (Figure 1), with a number of jewelry deigns with even higher numbers of variations.

Fretwork is a decorative design that is either carved in low relief on a solid background, or cut out to adorn a jewelry item. Fretworked bangles are gold jewelry items adorned with fretwork designs. In particular, this paper makes the technical contribution of developing a parametric feature based jewelry modeler for designing and manufacturing of the Fret-worked bangles.
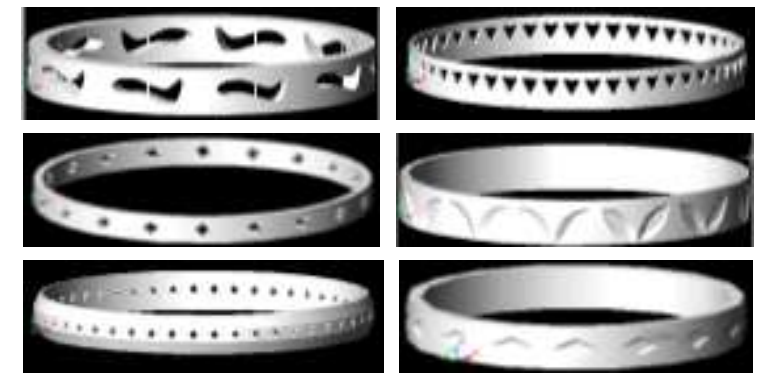

Figure 1: Fret-worked bangles

The developed jewelry modeler is a basic parametric feature based CAD paradigm. Parametric modeling is incorporated to capture the designer's intent, to get the variation in jewelry designs and to support customized jewelry design process. Also, by parameterizing the process, it is very easy to modify characteristics of the jewelry such as the size and the shape. In feature based design, features and stock-solids are the modeling components for which user define the key parameters. Features are placed to various faces of a stock-solid and desired product model can be built by applying the appropriate modeling operators such as the Boolean set operators. In the modeler, the fretwork designs are viewed as user defined features that are to be recurrently removed from the stock-solid bangles.

The modeler is capable of producing real and tangible artifacts directly from 3-D solid CAD models. CAD/CAM technologies are used collaboratively from jewelry designing to fabricating stage. The jewelry modeler supports diverse integrated manufacturing through computer controlled manufacturing tools. Rapid prototyping technologies are increasingly being used in personnel embellishment, especially jewelry [1-3]. It can produce master pieces of intricate form of jewelry items from solid models. Lasers with the aid of CNC can also implement fretwork into flat and even 3-D gold jewelry types.

The following parametric feature based commercial CAD systems have been developed for the purpose of jewelry designing: (i) Matrix (ii) Rhinoceros (iii) Jewel CAD (iv) Tech Jams (v) Art CAM Jewel Smith (vi) Jewel Space (vii) CAD Jewel 3D [4-7]. All of these systems have capabilities of good rendering and exporting models to rapid prototyping machines. But in majority of these systems, designing is performed manually using various tools and usually the design steps cannot be programmed to be executed automatically. This means that each different piece of jewelry would have to be created basically from the beginning.

Some automated parametric systems have been developed where the jewelry design is expressed by a set of parameters and constraints and the user's participation in the design process is through the dentition of the parameter values. ByzantineCAD has been developed for the designing of pierced medieval Byzantine jewelry [8-9]. A parametric voxel based approach has been presented to produce for stretch formed jewelry [10]. A unified jewelry modeler has been created for designing and creating carved jewelry of sophisticated craftsmanship [11]. A feature based CAD approach to re-engineering jewelry has also been presented [12-13]. These approaches are aiming to produce robust and accurate models that can either be manufactured or modified to create new jewelry pieces. 


\section{STRUCTURE OF THE MODELER}

The purpose of creating a jewelry modeler is to eliminate the formal designer and to provide the design tool in the hand of the user. The user participates in the modeling process through the definition of parameter values that include geometrical, dimensional and location attributes.

At conceptualization level of the design process, jewelry class, style, type, size, and making process are decided as design factors (Table 1). The idea of symmetry is incorporated in the jewelry style to minimize the verbosity of the geometrical definitions. Therefore, bangles with only recurrent fretwork designs are considered.

Table 1: Jewelry design factors

\begin{tabular}{|c|c|c|c|c|}
\hline Class & Style & Type & Size & $\begin{array}{c}\text { Making } \\
\text { process }\end{array}$ \\
\hline $\begin{array}{c}\text { Precious } \\
\text { gold } \\
\text { jewelry }\end{array}$ & $\begin{array}{c}\text { Fret- } \\
\text { worked }\end{array}$ & Bangles & $\begin{array}{c}\text { Wrist of } \\
\text { arm }\end{array}$ & $\begin{array}{c}\text { Investment } \\
\text { casting }\end{array}$ \\
\hline
\end{tabular}

A library of modeling components is created for the Fret-worked bangles. First type of modeling component is the basic constructive stock-solids in the form of bangles. Another type is the fretwork designs, which are viewed as a volume to be recurrently removed from the stock-solid. Different profiles of the solid bangles and fretwork designs are defined with their dimensional parameters and constraints. The variations in the jewelry design models can be obtained either by changing the modeling parameters or by changing the modeling components.

The final piece of jewelry type is produced by applying a sequence of acts. The user interacts with the modeler by choosing and then defining modeling parameters for the modeling components. The modeler creates an instance of solid bangle and then positions another instance of fretwork designs on the surface of the bangle for either carving or cutting of the designs. Once the rendering of custom designed jewelry is approved, the 3-D model is sent to the rapid prototyping machine for producing a master piece. A jeweler can produce casting from the master piece and bring the piece of custom made jewelry to a bright polish.

\section{MODELING COMPONENTS}

Two types of components have been created for modeling of the Fret-worked bangles; Stock-solids in form of the bangles and fretwork designs. These components are created using the sketch- based modeling approach. The modeling strategy starts with considering a plane from the three reference planes (XY, $\mathrm{YZ}$ and $\mathrm{ZX}$ ) for drawing a sketch. The sketch plane controls the orientation of a sketch in the 3-D modeling space. The sketches have been drawn using the 2-D comprehensive entities (line, arc, curve, circle, polygon, etc). Then, the sketch is swept for producing the modeling components. The stock-solids and fretwork designs make use of the rotational and translational sweeps respectively.

The bangles are used for personnel embellishment. The profile of inner face of the bangles, which touches the wrist of arm, would be flat to give comfort to the user. The outer face of the bangles, which adds artifacts to the bangles, would be generated with different profiles. Different sketches that form the profiles of outer face of the bangles, have been defined (Table 2) with their dimensional parameters and constraints. The simplest sketch is a rectangle (dimensions $\mathrm{b}$ and $\mathrm{z} ; \mathrm{b}>>\mathrm{z}$ ), which creates the solid bangle of width $b$ and thickness $t$ equal to $z$. Other sketches are drawn by changing the profile of a side of the rectangle. All these sketches are defined with the basic dimensions ( $b$ and $z$ ) and variants $\left(\mathrm{v}_{1}, \mathrm{v}_{2}, \mathrm{v}_{3}\right.$ and $\left.\mathrm{v}_{4}\right)$ and considered an axis symmetrical to minimize the verbosity of geometrical definitions. These variants are constrained within the values of the basic dimensions. The size (s) of the bangle, which suits the wrist of the arm of the user, is the distance between the inner side of the sketch and the revolved axis.

Table 2: The modeling component: Stock-solid

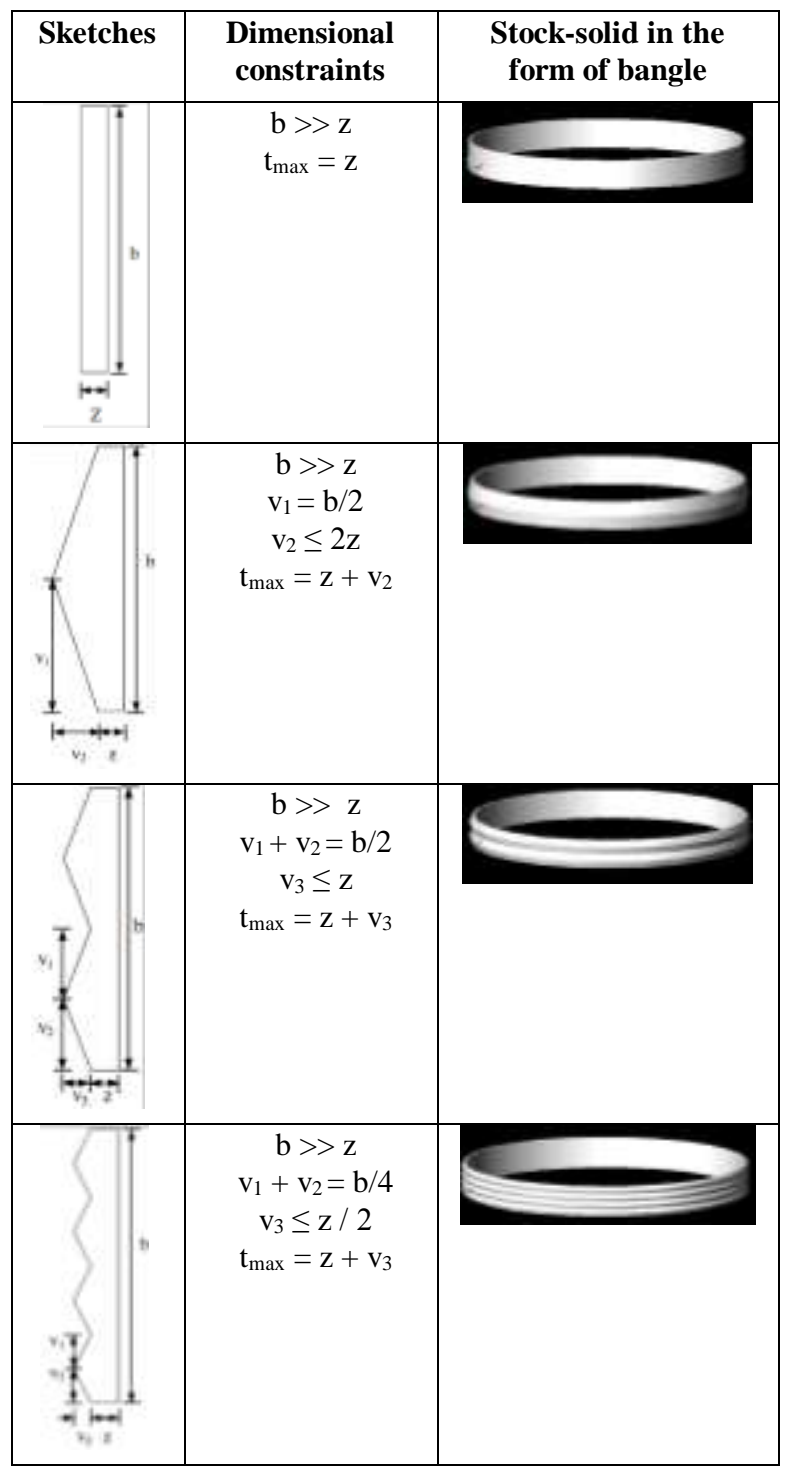




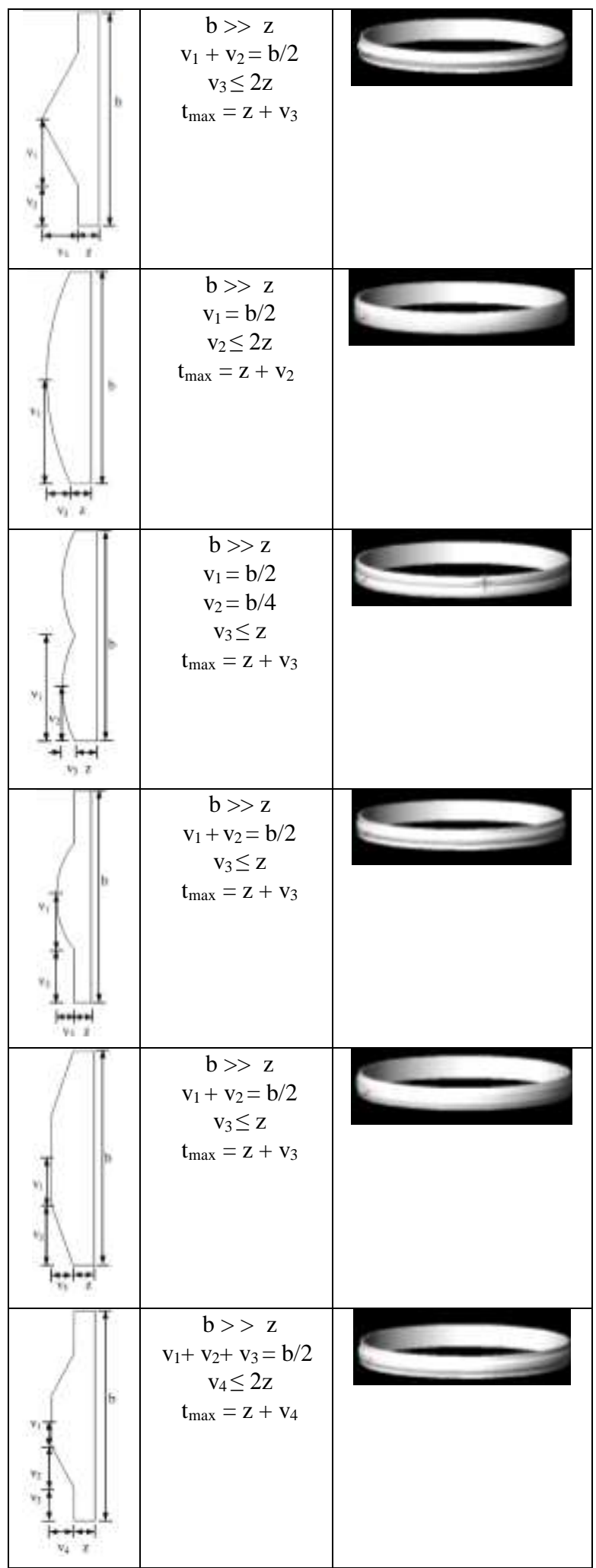

Fretwork designs are the features that are to be removed from the stock-solids. These designs are recurrently pierced or carved as a pattern on the surface of the solid bangles. Fretwork designs are the geometrical and floral sketches defined with dimensional parameters and constraints and shown in Table 3 (basic geometric shapes, regular polygons, diamond like shapes, arches, polygon stars or star like shapes, leaves, heart shape and flowers). These sketches use 2-D entities like lines, circles, polygons, arcs and curves. The catalogue of these fretwork designs is an open-ended list to which new definitions can be added to support custom jewelry modeling.

There exist different dimensional definitions in association with the different kinds of sketches. So, to reduce the dimensional definitions, fretwork designs are assumed to be inscribed in the circle or rectangle. The dimensions of the bounded circle $(\mathrm{R})$ or rectangle (B and $\mathrm{L}$ ) depend upon the dimensions of the stocksolid (b, t and s) and are taken less than the width (b) of the stock-solid. Recurrent number of fretwork designs as a pattern can be approximated by dividing the circumference $(2 \pi \mathrm{s})$ of the stock-solid with the length $(\mathrm{L})$ of bounded rectangle or diameter (2R) of circumscribed circle. The arbitrary center point of bounded geometries is used to position the fretwork design on the outer face of the stock-solid. The depth of the fretwork design is kept equal or less than the thickness of the stock solid (t) for fret working.

Table 3: The modeling component: Fretwork designs

\begin{tabular}{|l|l|}
\hline & Dimensional constraints \\
\hline & \\
\hline
\end{tabular}




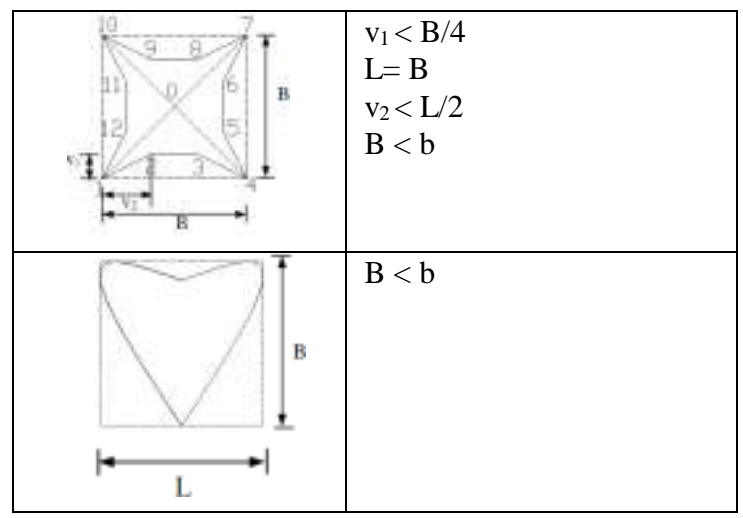

\section{MODELING PARAMETERS}

A variety of Fret-worked jewelry bangles can be modeled with the appropriate definitions of the following modeling parameters.

- The width (b) and thickness ( $t$ ) of the solid bangle derived from the dimensions ( $\mathrm{b}, \mathrm{z}$ and variants) of the sketches.

- The size (s) of solid bangle equal to the sweeping parameter that is distance between inner face of the sketch and the revolved axis.

- The size of the fretwork designs derived from the dimensions of bounded rectangle $(\mathrm{B}, \mathrm{L})$ or circle $(\mathrm{R})$ around the sketch for a class of the fretwork design.

- The different parameters $\left(\mathrm{v}_{1}, \mathrm{v}_{2}, \mathrm{v}_{3} \mathrm{p}, \mathrm{n}, \theta\right.$ in Table 3$)$ in association with the different classes of the fretwork designs.

- Position parameter of fretwork design to place on the surface of the stock-solid.

- Number of the fretwork designs to be placed recurrently as a pattern on the bangle. This parameter describes the intensity of fret-working.

\section{MODELING PROCESS}

The modeling process is completed in following steps:

- Select a stock-solid of desired shape and size in the front sketch plane (Figure 2a).

- Move the stock- solid to the side sketch plane (Figure 2b).

- Select a fretwork design of the desired class and position it on the outer face of the stock-solid such that arbitrary center point lies on line joining points $(0,0, s)$ and $(0, b, s)$ (Figure $2 \mathrm{c}$ ).

- Move to the top sketch plane and create multiple copies of fretwork designs about a point (s, 0, 0) (Figure 3d).

- Subtract the fretwork designs from the stock-solid (Figure 3e).

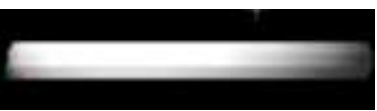

(a)

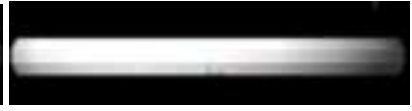

(b)

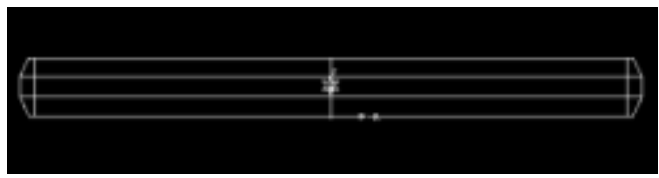

(c)

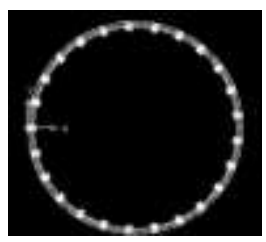

(d)

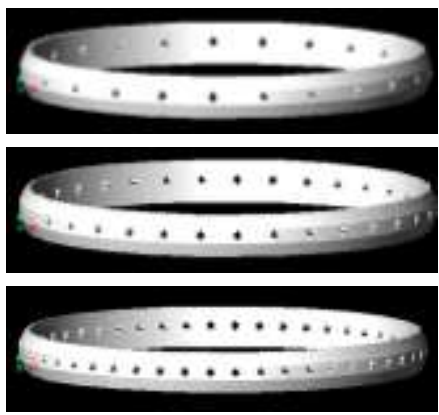

(e)

Figure 2: (a) The stock-solid in the front sketch plane (b) The stock-solid in the side sketch plane (c) Placement of the fretwork designs in the side sketch plane (d) Creation of multiple copies of the fretwork designs in the top sketch plane (e) The

Fret-worked bangles with variations in the number of the recurrent fretwork designs

\section{IMPLEMENTATION}

This paradigm is implemented under the ActiveX and Visual Basic Application (VBA) programming environment using AutoCAD. VBA runs in the same process space as AutoCAD, providing an intelligent and very fast programming AutoCAD environment. VBA sends messages to AutoCAD by the AutoCAD ActiveX Automation interface. AutoCAD VBA permits the VBA environment to run simultaneously with AutoCAD and provides programmable control of AutoCAD through the ActiveX Automation interface. AutoCAD is capable of creating STL file, which can be submitted to rapid prototyping machine which slices the design model into thin layers. Rapid prototyping process lays the cross sections layer by layer and combines the layers to create the jewelry pattern. CAD and rapid prototyping model of generated a Fret-worked bangle is shown in the Figure. 3.

\section{CONCLUSION}

The goal of generating a jewelry modeler for the Fret-worked bangles has been achieved through a basic parametric feature based CAD paradigm. This paradigm provides the capability of designing custom jewelry using the parametric design concept that gives a large number of variations in the designs. This work is in the direction to provide the design tool in the hand of actual users. Standardization as well as automation has been included through the integration of CAD-CAM tools. The geometric modeling techniques used in this work are quite simple, yet their results are very beautiful and inspiring. 


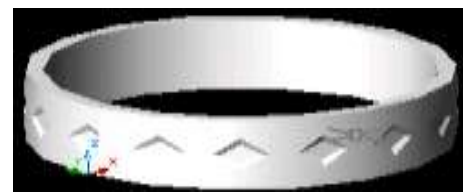

(a)

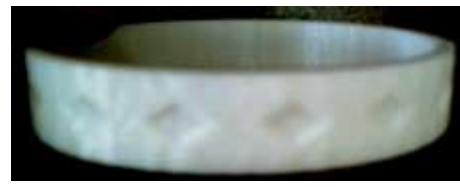

(b)

Figure 3: CAD and rapid prototyping model of a Fret-worked bangle

\section{REFERENCES}

[1] Molinari L. C. and Megazanni M. C., 1998, The ole of $\mathrm{CAD} / \mathrm{CAM}$ in the Modern Jewelry Business, Gold Technology, Vol. 23, pp. 3-7.

[2] Molinari L. C. and Megazanni M. C., 1996 Rapid Prototyping: Application to Gold Jewelry production, Gold Technology, Vol. 20, pp.10-17.

[3] Wannarumon S. and Bohez E. L. J., 2004, Rapid Prototyping and Tooling Technology in Jewelry CAD, Computer-Aided Design \& Applications, Vol. 1, No. 1-4, pp. 569-575.

[4] ArtCAM Jewel Smith, http://www.artcamjewelsmith.com, Delcam plc.
[5] TechGems 3.0, http://www.techjewel.com, TechJewel.

[6] JewelSpace, http://www.jewelspace.net, Caligory Software.

[7] JewelCAD, http://www.jcadcam.com, Jewelry CAD/CAM Ltd.

[8] Stamati V. and Fudas, I., 2005, A Parametric Feature Based CAD System for Reproducing Traditional Pierced Jewelry, Computer Aided Design, Vol. 4, No. 37, pp. 431-449.

[9] Stamati V., Fudas I., Theodoridou, S., Edipidi C. and Avramidis D., 2004, Using Poxels for Reproducing Traditional Byzantine Jewelry, Computer Graphics International 2004, Crete, Greece, June 16-19.

[10] Gulati V. and Tandon P., 2007, A Parametric Voxel Oriented CAD Paradigm to Produce Forming Components for Stretch Formed Jewelry, Computer Aided Design \& Applications, Vol. 4, No. 1-4, pp.137-145.

[11] Gulati V., Singh H. and Tandon P., 2008, A Parametric Voxel Based Unified Modeler for Creating Carved Jewelry, Computer Aided Design \& Applications, Vol. 5, No. 6, pp.811-821.

[12] Stamati V. and Fudas, I., 2005, A Feature-Based CAD Approach to Jewelry Re-Engineering, Computer-Aided Design \& Applications, Vol. 2, Nos. 1-6, pp. 1-9.

[13] Fudos I., 2006, CAD/CAM Methods for Reverse Engineering: A Case Study of Re-engineering Jewelry, Computer-Aided Design \& Applications, Vol. 3, No 6, pp. 683-700. 